\title{
Intervenção do Estado e desenvolvimento local: uma análise cross section dos municípios paranaenses
}

The State intervention and local development: the cross-section analyze of the paranaenses municipalities

Intervention de l'État et développement locale: une analyse cross section des villes paranaenses

Intervención del Estado y del desarrollo local: una sección representativa del análisis de las ciudades paranaenses

\author{
Christian Luiz da Silva* \\ Carminda Lopes** \\ William Michon Junior***
}

Recebido em 24/09/2008; revisado e aprovado em 23/12/2008; aceito em 02/02/2009.

\begin{abstract}
Resumo: A intervenção do Estado tem o propósito de fomentar o desenvolvimento local. O objetivo deste artigo é discutir essa capacidade de intervenção para a melhora das condições de desigualdade no Estado Paraná, mediante uma análise cross section dos municípios paranaenses. A pesquisa foi aplicada, descritiva e quantitativa, com análise dos dados para o ano de 2000. O estudo evidencia que as finanças públicas ainda não representam um papel efetivo para mudança das desigualdades socioeconômicas, mas afetam positivamente os indicadores sociais.

Palavras-chave : Desenvolvimento local. Municípios. Paraná. Finanças Públicas.
\end{abstract}

\begin{abstract}
The intervention of the State has the intention to foment the development local. The objective of this article is to argue this capacity of intervention for the improvement of the conditions of inequality in the Paraná State, by means of an analysis cross section of the Paraná cities. The research was applied, descriptive and quantitative, with analysis of the data for the year of 2000. The study it evidences that the public finances not yet represent an effective paper for change of the socioeconomics inequalities, but affect the social pointers positively.
\end{abstract}

Key words: Local development. Municipalities. Paraná. Public finances.

Résumé: L'intervention de l'État a l'intention de fomenter le développement local. L'objectif de cet article est discuter cette capacité d'intervention pour l'amélioration des conditions d'inégalité dans l'État Paraná, moyennant une analyse cross section des villes paranaenses. La recherche a été appliquée, descriptive et quantitative, avec analyse des données pour l'année de 2000. L'étude il prouve que les finances publiques ne représentent encore pas un papier efficace pour changement des inégalités socioeconômique, mais touchent positivement les indicateurs sociaux.

Mots-clé: Développement locale. Villes. Paraná. Finances Publiques.

Resumen: La intervención del estado tiene la intención de fomentar al local del desarrollo. El objetivo de este artículo es discutir esta capacidad de la intervención para la mejora de las condiciones de la desigualdad en el estado de Paraná, por medio de una sección representativa del análisis de las ciudades de los paranaenses. La investigación era aplicada, descriptiva y cuantitativa, con el análisis de los datos por el año de 2000. El estudio evidencia que las finanzas del público no todavía para representar un papel eficaz para el cambio de los inaqualities de los socioeconômicas, sino afectar los indicadores sociales positivamente.

Palabras clave : Desarrollo local. Ciudades. Paraná. Finanzas del Público.

\section{Introdução}

Os modelos de desenvolvimento local com intervenção do Estado pressupõem que a sua ação e influencia na orientação de elementos afetam positivamente no curto e no longo prazo a dinâmica da região. Entre os discursos políticos, saúde, educação e atração de empregos e investimentos são ações determinantes para reduzir a desigualdade social, violência e melhora da qualidade. Pode-se exemplificar a materialização destes

\footnotetext{
* Economista, Pós-doutor em Administração pela USP, professor do PPGTU/ PUCPR e da UTFPr. Pesquisador do $\mathrm{CNPq}$ - projeto de pesquisa intitulado: "Modelo dinâmico de desenvolvimento local para o apoio ao Planejamento Público" (processo 40085/2006-3)

** Economista e especialista em gestão econômica de negócios pela UniFAE Centro Universitário Franciscano do Paraná. Foi bolsista de iniciação científica da UNIFAE vinculada ao projeto "Modelo dinâmico de desenvolvimento local para o apoio ao Planejamento Público" (processo 40085/2006-3). E-mail: carmenlopes401@hotmail.com

*** Economista pela UniFAE Centro Universitário Franciscano do Paraná. Foi pesquisador da UNIFAE vinculada ao projeto "Modelo dinâmico de desenvolvimento local para o apoio ao Planejamento Público" (processo 40085/ 2006-3).E-mail: junior_wmjr@yahoo.ca
} 
discursos nos Planos Plurianuais do governo do Estado do Paraná de 2008 a 2011 (SEPL, 2007) ou o Plano de Governo de 2003 a 2006 deste Estado (SEPL, 2003). A intervenção do Estado Federal, responsável pela receita maior que $1 / 3$ do PIB brasileiro (IPEADATA, 2008), distribuído entre governos estaduais e municipais, se transforma em uma importante injeção de recurso para concretizar as ações consideradas fundamentais para o processo de desenvolvimento, contudo as disparidades regionais permanecem e se consolidam, em alguns casos.

O Paraná apresenta a situação mais desigual entre os municípios se comparado aos demais Estados do sul e sua conformação ocorreu pela construção e colonização das diferentes regiões do Estado, cuja história recente da maioria dos municípios, menos de 60 anos, e dependente de ciclos de exploração de recursos naturais demonstra quanto foi impreciso o modelo de descentralização do Estado paranaense.

Alguns estudos recentes foram desenvolvidos, principalmente pelo Ipardes (Instituto Paranaense de Desenvolvimento Econômico e Social), sobre o diagnóstico da situação socioeconômico e ambiental do Paraná (IPARDES, 2003 e 2004), entretanto o enfoque desse artigo é avaliar se a intervenção do Estado vem contribuindo para reduzir essas disparidades. Cabe questionar, desta forma, se o problema é a falta de recursos, a eficiência ou eficácia da aplicação dos recursos que tornam pouco efetivo o processo de desenvolvimento no Paraná. Considerando que este processo está vinculado a forma de intervenção do Estado, parte-se da hipótese que não se trata de falta de recursos, mas de uma alocação essencialmente pouco eficaz, que torna o resultado sem efetividade para retomar um processo de desenvolvimento por todo Estado. A Lei de Responsabilidade Fiscal contribui para acompanhamento da eficiência dos recursos, mas não há um instrumento que permita avaliar os resultados.

Neste contexto, cabe discutir a capacidade de intervenção do Estado para melhora das condições de desigualdade no Estado do Paraná. Em parte pode-se argumentar que o Estado não reduziu a desigualdade socioeconômica no Paraná, mas não permitiu que se aprofundasse. Contra-argumen- tado, o presente artigo objetiva avaliar a efetividade da intervenção do Estado, por meio dos governos municipais, no processo de desenvolvimento local. Para isso será feito uma análise cross section com dados dos municípios para o ano de 2000, relacionando indicadores socioeconômicos com informações de receita e despesas municipais. Partese do princípio que se houver uma relação entre a alocação do recurso e o indicador resultante daquela ação será considerado que há efetividade na ação pública. Caso não haja a hipótese será validada e será justificado que falta avaliação da eficácia da alocação do recurso público para se avaliar e aprimorar a efetiva intervenção do Estado no processo de desenvolvimento. A metodologia será exposta na terceira seção, contudo cabe destacar que não se trata de avaliar um município específico e a evolução das ações municipais ao longo do tempo, mas especificamente de correlacionar as diferentes realidades municipais em termos de finanças públicas com a situação socioeconômica. Se os municípios com melhor condição financeira tiverem melhores indicadores comprova-se que a questão é a falta de recursos. Se isso não se confirmar, valida-se a hipótese, como salientado.

de desenvolvimento. A terceira seção apresenta a metodologia e a quarta seção analisa os resultados para o Estado do Paraná, considerando a situação dos municípios em 2000. A quinta seção evidencia as conclusões e propõe novos estudos.

\section{Intervenção do Estado no processo de desenvolvimento local: abordagem teórica}

A intervenção estatal pode acontecer de diversas formas na economia. Silva (2005a, p. 89-92) apoiado no Relatório do Banco Mundial (1997, p. 4) cita que o Estado tem cinco tarefas fundamentais e, portanto, funções de intervenção: formar uma base jurídica; manter políticas não-distorcionistas, inclusive a estabilidade macroeconômica; investir em serviços básicos e infra-estrutura; proteger os grupos vulneráveis; proteger o meio ambiente. Iglesias (2006) ainda inclui o importante papel para o Estado no desenvolvimento tecnológico e de inovações, bem 
como para promover consensos nacionais. Além disso, essas tarefas devem ser executadas com a eficiência e eficácia da gestão pública a fim de traduzir a melhor qualitativa da prestação de serviços aos cidadãos (LERDA, ACQUATELLA e GÓMEZ, 2005).

Como expõe Frey a intervenção estatal está presente nos discursos contemporâneos do desenvolvimento. $\mathrm{O}$ autor ressalta isso ao afirmar que "a intervenção estatal, via planejamento, é considerada indispensável para reduzir ou evitar os efeitos nocivos dos processos de crescimento econômico, ou ainda, para poder eliminar ou reparar distúrbios e danos já existentes" (2001, p. 7).

A intervenção do Estado está atrelada à aplicação de políticas públicas, e mensurar esta correlação passa a ser prioridade para estabelecer eficiência no direcionamento dos recursos públicos, que crescem proporcionalmente menos que as demandas por serviços públicos. As políticas públicas atendem a questões que vão além da visão unidimensional, ou seja, uma ação com foco na saúde pode afetar também a questão econômica e social (SILVA, 2005b).

Para acompanhar essas políticas, as quais têm influência em mais de uma dimensão, há que se ter meios de avaliar sua eficiência. Souza (2007) faz uma exaustiva discussão sobre o conceito de políticas públicas e sintetiza com o conceito de Lowi apud Rezende (2004, p. 13) onde se define como "uma regra formulada por alguma autoridade governamental que expressa uma intenção de influenciar, alterar, regular, o comportamento individual ou coletivo através do uso de sanções positivas ou negativas". Ainda para Lowi (1972) a política pública pode assumir quatro formatos: distributiva, regulatória, redistributiva e constitutiva. Cada uma dessas políticas gera pontos ou grupos de vetos e de apoios diferentes, processando-se localmente de forma diferente. O objeto de discussão desse artigo é como essas políticas, em funções diferentes a partir do exercício da administração pública municipal no Paraná, podem ter contribuído para melhora efetiva dos indicadores socioeconômicos municipais.

O impacto do investimento público sobre a renda per capita (efeito direto sobre o produto) e sobre a produtividade total dos fatores (efeito indireto sobre o produto) é controverso. Cândido Junior (2006) estudou a Argentina, o Brasil e o Chile, com informações de 1970 a 2000, e concluiu que, para a amostra estudada, há uma relação positiva entre investimento público e produto. Contudo, a exceção do Chile, houve uma relação inversa entre investimento e produtividade. $\mathrm{O}$ autor se referiu a esta diferença como sendo a eficiência do uso dos recursos.

Cândido Junior (2006), contudo, acresceu, em sua análise, não somente a injeção de recursos, como a necessidade de avaliar sua eficiência. A conclusão a favor do Chile, na relação positiva do investimento público tanto em termos do produto quanto da produtividade, foi explicada, pelo autor, como a eficácia dos investimentos públicos. Neste sentido, um investimento eficaz produziria um efeito direto positivo na economia e indireto por meio do aumento da produtividade. Monteiro Neto (2006) não discute a eficiência do investimento público, mas o considera "um dos elementos mais importantes para a ativação da formação de capital na economia brasileira da última metade do século XX" (2006, p. 24). O autor complementa afirmando que "há elementos indicativos de que as reduções no gasto em investimento governamental, entre os períodos de 1991-1995 e 1996-2000, tiveram efeitos propagadores sobre a queda no nível geral de atividade mais que proporcionais ao que se poderia esperar" (MONTEIRO NETO, 2006, p. 26). Contudo, pondera Monteiro Neto, em consonância com Prichett (2000), ao afirmar que a intervenção estatal não pode ser vista como uma panacéia para a resolução de todos os problemas e da economia brasileira.

Pritchett (2000) já alertava sobre o uso indiscriminado do investimento público como panacéia da eficiência e da resolução dos problemas. Ao estudar essa questão, Oliveira (2006, p. 275) argumenta que "um dos motivos que leva a falhas nos resultados públicos é a dissociação que se faz entre elaboração e implementação no processo de planejamento de acordo com algumas visões da prática ou escolas de pensamento". O autor cita outro trabalho de sua autoria para justificar as falhas de políticas públicas em desenvolvimento por motivos político 
institucional, financeiros e técnicos (OLIVEIRA, 2002).

O primeiro motivo se refere a articulação e funcionamento do sistema político e da interação entre Estado e sociedade civil. $\mathrm{O}$ amadurecimento das bases institucionais depende de processos específicos e históricos em cada país e é um condicionante importante para garantir a implementação de políticas públicas. A questão financeira, segundo o autor, é "a razão mais direta para explicar as falhas em planejamento nos países em desenvolvimento" (OLIVEIRA, 2006, p. 280). A falta de recursos inibe qualquer possibilidade de implementar as políticas públicas da forma planejada, o que dificulta sobremaneira para alcançar resultados efetivos. Menicucci (2007) exemplifica esse problema ao discutir a questão da implementação da reforma sanitária.

Por fim, a capacidade técnica se refere a recursos humanos capacitados e motivados, equipamentos, experiência e competência técnica dos órgãos responsáveis para planejar e implementar as políticas públicas. Com pesos diferentes, os tópicos sintetizados por essa capacidade técnica são responsáveis de alguma forma para a ineficiência da implementação das políticas públicas. Às vezes é a falta de recursos humanos o mais preeminente, outras são equipamentos.

A avaliação da eficiência do investimento público poderia ser feita sob quatro óticas: eficiência na avaliação prévia da política pública; na interação entre os agentes que administram a política; na capacidade técnica para implementação; e no resultado, relacionando o proposto com o alcançado. Cada uma delas se refere a uma etapa do projeto. A primeira seria avaliada quanto a concepção (estágio inicial); a segunda se refere a etapa intermediária entre a concepção e a imple-mentação; a terceira se refere a implementação e a quarta ao resultado. Caso a concepção ocorra no mesmo âmbito da implementação (agente público local, por exemplo) haveria apenas as duas últimas fases para avaliação. Este artigo considera esta hipótese e avalia somente a última fase (ver metodologia na seção 3). As dificuldades de uso da avaliação das políticas públicas para melhorar a efetividade do uso dos recursos públicos é discorrida por Faria e
Filgueiras (2007) e exemplificado para o caso dos sistemas de avaliação. Assim, as alternativas de uso das avaliações tornam a política pública retroalimentadas e redirecionada para sua efetividade, quando utilizada de forma educativa.

Neste sentido Dubresson e Fauré (2005) convidam a repensar, também, sobre a freqüente relação entre descentralização e desenvolvimento local tida como mecânica. Ao estudar regiões da África do Sul, Brasil, México, Índia e Senegal os autores demonstram que existem desajustes entre os organismos institucionais de descentralização e o nível de formação ou de consolidação das dinâmicas econômicas locais.

A interação entre capacidade técnica e ambiente institucional pode ser verificada, desta forma, na relação entre Estado maior e as instituições locais. Adicionalmente a ineficiência de intervenções estatais pela falta de sintonia entre esferas estatais e realidades locais, pode haver a incapacidade técnica para implementação do projeto. Algumas situações podem colaborar com essa afirmação, quando, por exemplo, o planejamento ocorre centralizado, sem sintonia com a realidade local, que deve efetivamente implementar o projeto. $\mathrm{O}$ insucesso decorre da falta de habilidade para desenvolver o que é mais próximo ao desejado e pela baixa sincronia entre o Estado e as instituições locais. Brakely (1994, p. 41) avalia as opções de políticas nacionais e desenvolvimento e argumenta que as políticas nacionais seriam mais efetivas se acoplada com uma ativa política regional que garantisse a implementação de programas e desse suporte as atividades do desenvolvimento econômico. A eficiência desta implementação aconteceria, portanto, segundo o autor, se houvesse integração entre o poder local e nacional.

A descentralização não é, contudo, um consenso. Froehlich (1998, 88-89), citando Mattos, argumenta que a descentralização respalda um discurso de melhoria da democracia social, a participação popular e a justiça social, com propósito de viabilizar o desenvolvimento regional. Contudo, para Mattos citado em Froehlich, esta retórica seria do interesse somente dos que defendem a reforma do Estado, visando aprimorar os processos de acumulação e crescimento do 
capital. Por outro lado, Blakely (1994, p. 49) menciona que o desenvolvimento local representa não apenas uma nova retórica, mas uma importante alternativa que associa as atividades econômicas com o desenvolvimento local. O autor não marginaliza a participação do Estado, mas ressalta a necessidade de uma ação oriunda dos poderes locais, sejam eles públicos ou privados.

Contudo, Rodrigues (2007) mostra, por meio de um estudo evolutivo dos gastos públicos catarinenses de diferentes municípios, que não há grandes variações do direcionamento da verba público entre os locais analisados, mesmo com forte divergência ideológica e partidária. $\mathrm{O}$ autor argumenta, sobre este fato, que isso decorre da não-liberdade de aplicação dos recursos, pois os destinos são previamente definidos, restando pouco a ser aplicado livremente.

Neste contexto cabe verificar até que ponto ações propositivas são possíveis por meio de políticas públicas para melhora dos indicadores socioeconômicos e se elas estão sendo efetivas ou não. Assim, a participação do Estado neste artigo será considerado por meio das finanças públicas e correlacionado com os indicadores socioeconômicos dos municípios do Estado a partir de corte temporal (2000). O modelo teórico pressupõe, portanto, que as finanças públicas representam uma importante forma de intervenção do Estado e são variáveis explicativas da redução das desigual-da-des socioeconômicas a partir da educação, saúde e emprego, explicadas para o processo de desenvolvimento. Antes de apresentar os resultados a próxima seção retrata a metodologia de pesquisa.

\section{Metodologia}

A hipótese desse artigo é que a efetividade da intervenção do Estado para redução da desigualdade socioeconômica do Estado é fruto de uma alocação essencialmente pouco eficaz, e não pela falta de recursos. A validação desta hipótese parte da avaliação da relação entre a alocação dos recursos públicos e os indicadores socioeconômicos resultantes daquela ação. Se houver correlação pressupõem-se haver efetividade na ação pública, caso contrário se valida a hipótese e se considera que a mesma depende de uma gestão mais eficaz dos recursos públicos. Para isso, fez-se uma análise cross section (corte temporal) do ano 2000 para todos os municípios paranaenses. A escolha do ano 2000 deve-se ao fato de se ter o maior número de variáveis para análise (última base censitária para todos os municípios).

Foram levantadas 95 variáveis e classificadas em 8 áreas: desigualdade socioeconômica, finanças públicas, educação, produção e consumo, infra-estrutura e demografia, emprego, renda e saúde, que foi subdividida em longevidade e saneamento. A listagem das variáveis e respectivas fontes se encontra no apêndice 1 . O quadro 1 apresenta a relação das áreas e da quantidade de variáveis envolvidas para todos os municípios paranaenses.

\begin{tabular}{|lc}
\hline \multicolumn{1}{|c}{ ÁREAS } & QUANTIDADE \\
DE VARIÁVEIS \\
Desigualdade socioeconômica & 3 \\
Educação & 8 \\
\hline Finanças Públicas & 20 \\
\hline Infra-estrutura e demografia & 11 \\
\hline Emprego & 6 \\
Produção e Consumo & 22 \\
Renda & 12 \\
Saúde (longevidade e saneamento) & 13 \\
\hline TOTAL & 95 \\
\hline
\end{tabular}

Quadro 1 - Quantidade de variáveis por área analisada para os municípios paranaense 2000

Fonte dos Dados Brutos: IBGE, PNUD, COPEL, IPEA, STN.

Nota: Ver lista de todas variáveis utilizadas no apêndice 1 .

A pesquisa realizada é aplicada já que depende dos conhecimentos desenvolvidos pela pesquisa pura, mas tem como interesse à aplicação e conseqüências práticas do conhecimento. A pesquisa pode ser classificada como descritiva no que se refere ao objetivo geral, pois busca a descrição das características de determinada população ou fenômeno a partir da utilização de técnicas padronizadas de coleta de dados. Trata-se também de uma pesquisa quantitativa, segundo a abordagem do problema, pois o enfoque é a coleta e tratamento de informações por meio de técnicas estatísticas, com corte temporal (cross section) para 2000, como já foi justificado, especialmente análise de corre- 
lação bivariada e análise de regressões para variáveis chaves, como educação, saúde, emprego e desigualdade (GIL, 2000; DIEHL e TATIM, 2004; BÊRNI, 2002).

As etapas de consecução da coleta e tratamento dos dados foram: 1) escolha das variáveis de dados disponíveis para o ano 2000 nas fontes indicadas, que são as mais representativas para informações municipais no Brasil; 2) coleta e estruturação das informações; 3) definição de indicadores compostos, conformados por variáveis diretas, como, por exemplo, despesas municipais per capita. As variáveis iniciais mais estas tratadas totalizaram 95 dados para este período e para todos os municípios. (Alguns municípios não tinham observações de alguns dados e neste caso foi atribuído valor nulo); 4) foi realizado a análise de correlação bivariada entre todas as variáveis; 5) destacou-se as correlações significativas (Correlação Pearson próximo de zero); 6) para essas correlações distribui-se entre correlações fortes (valores absolutos acima de 0,7), médias (entre 0,69 e 0,40 ) e fracas (menor que $0,4) .7$ ) avaliaram-se as variáveis explicativas e correlacionadas de cada área usualmente objeto de ações públicas, determinadas como sendo desigualdade, educação, saúde e emprego e, verificou-se a significância do sinal da correlação (positiva ou negativa) frente a teoria e o nível de correlação (forte, média ou fraca); 8) analisou-se os resultados com a finalidade de validar ou não a hipótese, apresentando-se inicialmente alguns indicadores das mesoregiões paranaenses a fim de mostrar as disparidades do Estado. Cabe salientar que toda análise estatística foi realizada com o suporte do software SPSS versão 12.0.

Algumas limitações metodológicas são pertinentes: primeiro, ressalta-se que a limitação de estudos a partir de modelos estatísticos e econométricos tradicionais, apontado por Soto Torres e Fernandez Lechón (2006), referente a perda da componente histórica é uma limitação deste artigo também. Contudo, essas limitações são justificadas pelo propósito do trabalho. Não se pretende avaliar a evolução dos municípios, mas se a diferença de recursos públicos é suficiente para explicar a desigualdade socioeconômica. Validar essa hipótese abre campo para pesquisa mais qualitativa e histórica das diferenças de cada município, mas primeiro é importante ter a melhor definição do problema de pesquisa a partir deste estudo descritivo.

Outra limitação, associada a esta, é não considerar a diferença de eficácia da gestão entre os municípios, por não tratar da evolução histórica. Novamente, o propósito é identificar em termos gerais, sem, neste momento, entrar no mérito de cada município, que será fruto de novos trabalhos.

\section{Análise dos resultados}

A compreensão do processo de desenvolvimento paranaense origina-se nas diferentes formas de colonizações das regiões, tanto em termos de período histórico quanto motivação (MAGALHÃES FILHO, 1999). Desta forma, inicialmente serão apresentados alguns indicadores socioeconômicos que retratam essas disparidades para em seguida analisar a correlação desses indicadores, conforme a metodologia descrita anteriormente.

\subsection{Evolução do desenvolvimento paranaense recente}

Durante o processo de reconfiguração da estrutura produtiva na década de 1990 (SILVA e MICHON JUNIOR, 2008), os grandes centros passaram a ofertar mais empregos e a infra-estrutura destes centros atraiam a migração da zona rural para a zona urbana, em busca de melhor qualidade de vida, uma vez que eram ofertados serviços públicos que muitas vezes não eram ofertados na quantidade ou qualidade necessária nas zonas rurais. A tabela 1 apresenta a migração entre 1980 e 2000, ressaltando a migração ocorrida no Paraná da zona rural para urbana.

Tabela 1 - População residente urbana

\begin{tabular}{l|c|c|c|c}
\hline POPULACÃO URBANA & 1980 & 1991 & 1996 & 2000 \\
\hline BRASIL & $67,6 \%$ & $75,6 \%$ & $78,4 \%$ & $81,2 \%$ \\
BA - Bahia & $49.3 \%$ & $59.1 \%$ & $62.4 \%$ & $67.1 \%$ \\
MG - Minas Gerais & $67,1 \%$ & $74,9 \%$ & $78,4 \%$ & $82,0 \%$ \\
PR - Paraná & $58,6 \%$ & $73,4 \%$ & $77,9 \%$ & $81,4 \%$ \\
RJ - Rio de Janeiro & $91,8 \%$ & $95,3 \%$ & $95,5 \%$ & $96,0 \%$ \\
RS - Rio Grande do Sul & $67,5 \%$ & $76,6 \%$ & $78,7 \%$ & $81,6 \%$ \\
SC - Santa Catarina & $59.4 \%$ & $70.6 \%$ & $73.1 \%$ & $78.7 \%$ \\
SP - São Paulo & $88,6 \%$ & $92,8 \%$ & $93,1 \%$ & $93,4 \%$ \\
Demais Estados & $54,8 \%$ & $65,0 \%$ & $69,4 \%$ & $73,5 \%$ \\
\hline
\end{tabular}

Fonte: IPEADATA. 
No Paraná observou-se o mesmo processo entre as mesorregiões, no qual houve migração das mesorregiões menos industrializadas para os grandes centros. A tabela 2 mostra a dinâmica populacional entre 1980 e 2000. A região metropolitana de Curitiba cresceu $31 \%$ neste período, liderado pelo crescimento de $33 \%$ da população urbana. A população de outras regiões do estado migrou para a região urbana de RMC (Re- gião Metropolitana de Curitiba), a medida que a população urbana de outras regiões decresceu na sua participação na população urbana paranaense, enquanto a RMC apresentou o movimento inverso. O movimento da zona rural para a urbana ocorreu internamente no Paraná, principalmente no interior à medida que os postos de trabalham se abriam nas cidades.

Tabela 2 - Distribuição populacional no Paraná

\begin{tabular}{l|r|r|r|r}
\hline DISTRIBUICÁO POPULACIONAL & 1980 & 1991 & 1996 & 2000 \\
\hline Estado do Paraná & \multicolumn{4}{|c}{} \\
População & 7.629 .392 & 8.448 .713 & 9.003 .804 & 9.563 .458 \\
Part. \% População Estado & $100,0 \%$ & $100,0 \%$ & $100,0 \%$ & $100,0 \%$ \\
Grau de Urbanização & $58,6 \%$ & $73,4 \%$ & $77,9 \%$ & $81,4 \%$ \\
Part. \% População Urbana Estado & $100,0 \%$ & $100,0 \%$ & $100,0 \%$ & $100,0 \%$ \\
Metropolitana de Curitiba & & & & \\
População & 1.703 .787 & 2.319 .526 & 2.734 .500 & 3.053 .313 \\
Part. \% População Estado & $22,3 \%$ & $27,5 \%$ & $30,4 \%$ & $31,9 \%$ \\
Grau de Urbanização & $86,3 \%$ & $89,4 \%$ & $89,8 \%$ & $90,6 \%$ \\
Part. \% População Urbana Estado & $32,9 \%$ & $33,5 \%$ & $35,0 \%$ & $35,5 \%$ \\
Interior & & & & \\
População & 5.925 .605 & 6.129 .187 & 6.269 .304 & 6.510 .145 \\
Part. \% População Estado & $77,7 \%$ & $72,5 \%$ & $69,6 \%$ & $68,1 \%$ \\
Grau de Urbanização & $50,7 \%$ & $67,3 \%$ & $72,7 \%$ & $77,1 \%$ \\
Part. \% População Urbana Estado & $67,1 \%$ & $66,5 \%$ & $65,0 \%$ & $64,5 \%$ \\
\hline
\end{tabular}

Fonte: IPARDES.

A abertura da economia e o novo processo de industrialização demandaram ampliação da infra-estrutura, para atender o crescimento da produtividade e de novas máquinas utilizadas no processo de produção. A tabela 3 apresenta dados sobre o abastecimento de água e energia elétrica. O crescimento industrial pode ser apresentado a partir da proxy consumo de energia elétrica. Tanto a RMC quanto o interior apresentaram um crescimento elevado, pela maior disponibilidade de energia no estado, e pelo crescimento da demanda por energia. $\mathrm{O}$ crescimento da utilização da energia advém do uso mais intensivo da tecnologia tanto dos consumidores quanto do setor industrial, que pode ser visto pelo crescimento da razão consumo por consumidores nas localidades apresentadas.

No comparativo de consumo de energia elétrica brasileira com o Paraná, o consumo paranaense cresceu em escala superior ao brasileiro, em função do processo industrializante da década de 1990, que não se refletiu com tal grau de intensidade nos outros estados brasileiros que apresentam menor dinâmica industrial e que possuem maior dependência nos produtos primários ou pouco manufaturados. Enquanto o consumo brasileiro total e consumo brasileiro industrial cresceram $41,9 \%$ e $66,3 \%$ respectivamente, o Paraná experimentou um crescimento de $73,1 \%$ e $75,0 \%$ ao longo do mesmo período. 
Tabela 3 - Dados sobre a infra-estrutura paranaense 1990-2000

\begin{tabular}{|c|c|c|c|c|}
\hline INFRA-ESTRUTURA & 1990 & 1995 & 2000 & $\begin{array}{c}? \% \\
1990 \times 2000 \\
\end{array}$ \\
\hline \multicolumn{5}{|l|}{ Estado do Paraná } \\
\hline Abastecimento de Água - Ligações & 1.169 .279 & 1.574 .529 & 1.913 .973 & $63,7 \%$ \\
\hline Abastecimento de Água - Ligações Industriais & 7.037 & 8.404 & 9.302 & $32,2 \%$ \\
\hline Abastecimento de Água - Unidades Atendidas & 1.478 .727 & 1.948 .773 & 2.347 .877 & $58,8 \%$ \\
\hline Abastecimento de Água - Unidades Atendidas Industriais & 8.091 & 8.767 & 9.594 & $18,6 \%$ \\
\hline Energia Elétrica - Consumidores & 1.946 .241 & 2.466 .815 & 2.918 .680 & $50,0 \%$ \\
\hline Energia Elétrica - Consumo (mwh) & 9.767 .876 & 12.996 .213 & 17.093 .971 & $75,0 \%$ \\
\hline Energia Elétrica no Setor Secundário - Consumidores & 28.241 & 36.226 & 45.372 & $60,7 \%$ \\
\hline Energia Elétrica no Setor Secundário - Consumo (mwh) & 4.076 .554 & 5.350 .977 & 7.057 .546 & $73,1 \%$ \\
\hline \multicolumn{5}{|l|}{ Metropolitana de Curitiba } \\
\hline Abastecimento de Água - Ligações & 361.311 & 510.265 & 639.568 & $77,0 \%$ \\
\hline Abastecimento de Água - Ligações Industriais & 2.369 & 2.708 & 2.880 & $21,6 \%$ \\
\hline Abastecimento de Água - Unidades Atendidas & 516.910 & 702.829 & 872.139 & $68,7 \%$ \\
\hline Abastecimento de Água - Unidades Atendidas Industriais & 2.588 & 2.775 & 2.919 & $12,8 \%$ \\
\hline Energia Elétrica - Consumidores & 620.266 & 818.061 & 997.408 & $60,8 \%$ \\
\hline Energia Elétrica - Consumo (mwh) & 3.706.194 & 4.891 .816 & 6.662 .968 & $79,8 \%$ \\
\hline Energia Elétrica no Setor Secundário - Consumidores & 10.298 & 13.481 & 17.101 & $66,1 \%$ \\
\hline Energia Elétrica no Setor Secundário - Consumo (mwh) & 1.795 .586 & 2.202 .507 & 2.913 .437 & $62,3 \%$ \\
\hline \multicolumn{5}{|l|}{ Interior } \\
\hline Abastecimento de Água - Ligações & 807.968 & 1.064 .264 & 1.274 .405 & $57,7 \%$ \\
\hline Abastecimento de Água - Ligações Industriais & 4.668 & 5.696 & 6.422 & $37,6 \%$ \\
\hline Abastecimento de Água - Unidades Atendidas & 961.817 & 1.245 .944 & 1.475 .738 & $53,4 \%$ \\
\hline Abastecimento de Água - Unidades Atendidas Industriais & 5.503 & 5.992 & 6.675 & $21,3 \%$ \\
\hline Energia Elétrica - Consumidores & 1.325 .975 & 1.648 .754 & 1.921 .272 & $44,9 \%$ \\
\hline Energia Elétrica - Consumo (mwh) & 6.061 .682 & 8.104.397 & 10.431 .003 & $72,1 \%$ \\
\hline Energia Elétrica no Setor Secundário - Consumidores & 17.943 & 22.745 & 28.271 & $57,6 \%$ \\
\hline Energia Elétrica no Setor Secundário - Consumo (mwh) & 2.280 .968 & 3.148 .470 & 4.144.109 & $81,7 \%$ \\
\hline
\end{tabular}

Fonte: IPARDES.

Desta forma, houve incrementos significativos de recursos energéticos e aumento da rede de abastecimento de água. A energia é um indicador comumente utilizado como proxy para análise do grau de desenvolvimento de países e regiões, uma vez que quanto maior for o emprego e acessibilidade aos recursos tecnológicos, maior tende a ser a utilização de energia elétrica. Contudo, é possível haver incrementos tecnológicos que não representou incrementos diretamente proporcionais aos incrementos tecnológicos, uma vez que os aparelhos elétricos estão sendo projetados para poupar energia, aumentando sua eficiência na utilização do recurso, como por exemplo, a tecnologia empregada nas geladeiras nos últimos anos.

Partindo de tais premissas, foi possível identificar que houve incrementos significativos tanto na RMC quando no interior paranaense, demonstrando que o crescimento econômico atingiu todo o estado. A urbanização da população contribuiu mutuamente para tal fato, uma vez que se tratou de um sistema adaptativo positivo, pois a medida que as cidades se industrializavam, as pessoas se deslocavam do campo para a cidade em busca de oportunidades de trabalho. Enquanto a demanda por trabalho era maior que a oferta esta tendência mantevese ao longo do período analisado, contribuindo para o crescimento da demanda por energia elétrica.

Os processos de transformação da econômica brasileira e paranaense causaram efeitos na dimensão social, uma vez que foi identificado o processo de urbanização e desemprego ao longo do período. Portanto, nesta dimensão serão apresentados dados e indicadores que configuram os panoramas sociais, tais como da educação e violência, visando avaliar como as mudanças econômicas dos anos 1990 influenciaram as variáveis não-econômicas no decorrer desta década. Serão feitas algumas comparações entre os principais estados para situar a relevância das transformações.

As teorias mais recentes sobre desenvolvimento, sempre apontam a importância da educação para o processo de desenvolvimento no longo prazo. Somente a educação permite que a melhoria da produtividade seja continua sem causar impactos negativos no mercado de trabalho. A principal proxy utilizada para quantificar a variável educação é o anos de estudo da população acima de 25 anos. Segundo dados do IPEA, o Paraná foi um dos estados que mais aumen- 
taram os anos de estudo entre 1990 e 2001. Apesar de o Paraná estar acima da média da maioria dos estados, ainda há um gap entre o Paraná e os principais estados brasileiros, conforme mostra a tabela 4 .

Tabela 4 - Anos de estudo nos estados 19812001.

\begin{tabular}{l|c|c|c|c}
\hline \multicolumn{1}{c}{ ESTADOS } & \multicolumn{4}{c}{ ANOS DE ESTUDOS } \\
\cline { 2 - 5 } & 1981 & 1990 & 1995 & 2001 \\
\hline Distrito Federal & 6,3 & 7,5 & 7,7 & 8,2 \\
Minas Gerais & 3,6 & 4,6 & 4,9 & 5,7 \\
Paraná & 3,4 & 4,6 & 5,2 & 6,0 \\
Rio de Janeiro & 5,4 & 6,2 & 6,7 & 7,2 \\
Rio Grande do Sul & 4,3 & 5,3 & 5,8 & 6,3 \\
Santa Catarina & 3,9 & 5,0 & 5,6 & 6,4 \\
São Paulo & 4,7 & 5,7 & 6,1 & 6,9 \\
Demais Estados (Média) & 3,2 & 4,3 & 4,6 & 5,2 \\
\hline
\end{tabular}

Fonte: IPEA.

A distribuição de renda durante o período apresentou uma leve piora no índice de Gini em todos os estados brasileiros, resultante dos processos analisados anteriormente. A medida que o grau de urbanização cresceu, devido a migração das pessoas para os centros urbanos em busca de trabalho, e a produtividade das indústrias juntamente com competitividade do mercado, levou ao aumento do nível de desemprego da economia, influenciado negativamente pelas crises internacionais. No caso paranaense, o Índice de Gini passou de 0,597 para 0,607 entre 1991 a 2000, segundo dados do IPEA (Instituto de Pesquisa Econômica Aplicada), apesar de haver redução da pobreza entre 1991 e 2000 conforme dados do IPEA, o que indica que embora a população pobre e indigente esteja reduzindo, a população mais rica está crescendo na participação da renda apropriada da população. A violência medida a partir do indicador de taxa de homicídios por 100 mil habitantes, cresceu $28 \%$ no Paraná entre 1990 e 2000 de acordo com dados do IPEA, enquanto a média brasileira foi de $22 \%$ para o mesmo período.

Em síntese a história recente paranaense pode ser tratada pela elevação da desigualdade, devido ao desemprego e a maior concentração de renda pelos mais ricos, mantiveram o crescimento da violência analisada a partir da taxa de homicídio. Contudo, o maior grau de urbanização e a demanda por mão de obra qualificada melhoraram a educação da população, além da criação de novas universidades no estado e da alte- ração do sistema educacional do ensino fundamental, reduzindo a evasão escolar e aumentando a quantidade média de anos de estudos da população adulta, ou seja, houve uma melhora quantitativa na educação.

\subsection{Correlação das finanças públicas com indicadores socioeconômicos dos municípios paranaenses}

As variáveis componentes da análise envolveram a qualidade da educação, a mortalidade infantil, a desigualdade e a receita municipal. Avaliaram-se as variáveis explicativas e correlacionadas com estas a fim de compreender como a diferença de realidade entre os municípios paranaenses em 2000 justificava a variação das mesmas. As reflexões a seguir se referem aos dados apresentados nas tabelas A1 a A4 do apêndice.

Ao analisar a correlação das diversas variáveis a fim de compreender a importância e com capacidade explicativa da dinâmica dos municípios paranaense pode-se verificar que as variáveis são interdependentes, como se pretende explicar. Uma variável atinge a outro com a mesma força e sentido. Observou-se que o IDEB (Índice de Educação Básica), que mensura a qualidade do ensino básico, tem forte correlação com a mortalidade infantil, em sentido inverso (quanto melhor a qualidade do ensino menor é a mortalidade infantil, que traz mais saúde e melhora o desempenho escolar). O IDEB tem correlação positiva com o nível de estudo das pessoas com mais de 25 anos, o que significa que a educação básica com qualidade estimula as pessoas a darem valor a educação, que por usa vez impacta positivamente o IDH (Índice de Desenvolvimento Humano). Quanto maior a escolaridade, maior é o nível de desenvolvimento humano. Em sentido oposto, o IDEB tem uma correlação negativa com a taxa de pobreza (quanto mais pobres, menor o desempenho; e quanto menor o desempenho mais eles continuam pobres), mas positiva com a renda per capita (quanto maior o nível de escolaridade, maior a renda média per capita). Notou-se, contudo, que a participação pública, por meio das despesas públicas não é representativa e significativa para explicar o nível de desenvolvimento do IDEB. Os gastos públicos se 
relacionam mais com o formalismo burocrático, como está fazendo, e com a população em geral, significando que o problema dos recursos na educação não é quanto se gasta, mas como se gasta. Esse é um problema contundente para qualquer avaliação de política pública.

A saúde também mostra uma relação fraca com o gasto público da saúde, sendo que a educação, mensurada pelo IDEB, é mais representativa para explicar a mortalidade infantil que o gasto com saúde, corroborando com a hipótese acima. O grau de urbanização também influência positivamente a redução da mortalidade infantil pelo acesso aos domicílios com serviço de esgoto, água e lixo. A mortalidade infantil tem forte relação inversa com o IDH, com a expectativa de vida ao nascer, com a própria renda e com a educação. Quanto maior a mortalidade infantil, maior é a desigualdade mensurada pelo índice de Gini, maior é a pobreza e a taxa de fecundidade. Isso significa que a condição de vida do indivíduo conforma as necessidades vitais em termos de renda e saúde e retrata o nível da mortalidade infantil.

A desigualdade socioeconômica, mensurada pelo índice de Gini, diminui à medida que aumentam os anos de estudos, os domicílios com saneamento, a renda e o grau de urbanização. Os municípios mais urbanos são mais desiguais, quando viabilizam o acesso de domicílios com água encanada, esgoto e coleta de lixo é freqüente. Além disso, o tempo de estudo é uma variável importante para reduzir essa desigualdade. Em síntese, nesta etapa, pode-se evidenciar, a partir dos dados dos municípios paranaenses, como a educação com qualidade é um elemento crucial para buscar alternativas de desenvolvimento que sustentem um processo mais contínuo de oportunidades e novas ações.

A receita municipal, contudo, apresenta uma situação reativa, ou seja, ela é influenciada pela economia e não o contrário. Quanto maior o PIB, o emprego e o número de estabelecimentos maior será a receita do governo. Enquanto isso, o aumento da pobreza e a redução da renda per capita, não diminuem a desigualdade também. A receita será proveniente de uma dinâmica econômica que fortaleça a origem dos recursos.
Tais correlações permitem inferir que o maior problema relativo a efetividade da aplicação dos recursos públicos se refere a como é utilizado e não a falta dele. Por isso, há um entendimento equivocado entre o processo de orçamento e execução orçamentária e o planejamento estratégico municipal. O primeiro não substitui o outro, como alerta Rezende e Castor (2006). O planejamento estratégico municipal deve estabelecer as potencialidades e as dificuldades ao tratar do uso dos recursos públicos frente as demandas existentes, seguindo princípios de eficiência e eficácia dos recursos aplicados para atender essa demanda. Isso pode gerar a competição entre municípios, que é negativa no longo prazo pelos malefícios da concentração regional. Por isso, um plano estratégico municipal deve estar alinhado com uma visão regional e local. Contudo, cabe destacar alguns problemas de execução desse plano estratégico: 1) a administração dos recursos muda a cada 4 anos e, eventualmente, modifica o planejamento estratégico municipal, mesmo que não seja significativo; 2) as limitações legais para aplicação dos recursos (RODRIGUES, 2007); 3) visão de curto prazo em detrimento ao longo prazo e a visão municipal em detrimento a regional; 4) histórico fortemente construído a despeito da administração pública naquele momento (SOUZA, 1997; BLAKELY, 1994); 5) recursos humanos pré-determinados e não necessariamente com o perfil desejado pelo administrador (OLIVEIRA, 2006).

Superar esses problemas permitiria que o planejamento estratégico municipal fosse executado e as dificuldades oriundas da desigualdade regional sejam superadas e potencializadas as oportunidades, contudo esses problemas ainda estão distantes de serem resolvidos pela estrutura institucional do Estado no Brasil.

\section{Conclusões}

O objetivo desse artigo era avaliar a efetividade da intervenção do Estado, por meio dos governos municipais, no processo de desenvolvimento local e pode-se destacar a sua contribuição para academia, sociedade e administração pública. Para academia, traz uma discussão ampliada sobre a 
efetividade da intervenção do Estado, mostrando que no Paraná não houve uma contribuição efetiva das administrações municipais para reduzir as desigualdades. Para sociedade pode-se inferir justamente sobre as limitações da administração pública nesse processo e mostrar que não existe grande margem de manobra para aplicação dos recursos e uma preocupação excessiva com a eficiência e não com a eficácia dessa aplicação. Para a administração pública mostrouse a importância de um planejamento estratégico municipal e vinculo com as proposições regionais, não somente municipais, para buscar otimizar o uso dos recursos, mesmo com as limitações mencionadas.

Assim, cabe destacar que as finanças públicas ainda não representam um papel efetivo para mudança das desigualdades socioeconômicas dos municípios paranaenses. Verificou-se que há uma dinâmica própria em cada município a partir das oportunidades de desenvolvimento econômico local que incrementam as finanças públicas e afetam positivamente os indicadores sociais. Com isso, mostrou-se a necessidade de discutir a capacidade de intervenção do Estado. Não pela eficiência na alocação dos recursos, controlado pelas instâncias de fiscalização, mas pela eficácia da aplicação.

Por fim, propõem-se como novos trabalhos um estudo qualitativo de administrações públicas que conseguiram superar os problemas, estabelecer e implementar um planejamento estratégico municipal com efetiva mudança da desigualdade social.

\section{Referências}

BANCO MUNDIAL. Relatório sobre o Desenvolvimento Mundial 1997. Washington/USA, 1997.

BÊRNI, Duilio de Avila (coordenador). Técnicas de pesquisa em economia: transformando curiosidade em conhecimento. São Paulo: Saraiva, 2002. $\left(^{*}\right)$

BLACKELY, Edward J. Planning Local Economic Development: theory and practice. 2. ed. USA: Sage Publications, 1994.

CÂNDIDO JR, José Oswaldo. Efeitos do investimento público sobre o produto e a produtividade: uma análise empírica. Texto para discussão 1204. IPEA - Instituto de Pesquisa Econômica Aplicada, Brasília, agosto 2006.

DIEHL, Astor Antônio; TATIM, Denise Carvalho. Pesquisa em ciências sociais aplicadas. São Paulo: PrenticeHall/ Pearson Education, 2004. $\left(^{*}\right)$

DUBRESSON, Alain; FAURÉ, Yves-André.
Décentralisation et développement local: un lien à repenser. Revue Tiers Monde, n. 181, janvier-mars 2005.

FARIA, C.A.P.; FILGUEIRAS, C.A.C. As políticas dos sistemas de avaliação da educação básica do Chile e do Brasil. In: HOCHMAN, G.; ARRETCH, M.; MARQUES, E. (org.). Políticas públicas no Brasil. Rio de Janeiro: Fiocruz, 2007. p. 327-368.

FREY, Klaus. A dimensão político-democrática nas teorias de desenvolvimento sustentável e suas implicações para a gestão local. Ambiente $\mathcal{E}$ Sociedade, ano IV, n. 9, 2o semestre de 2001.

FROEHLICH, José Marcos. O "local” na atribuição de sentido ao desenvolvimento. Revista Paranaense de Desenvolvimento, Curitiba, n. 94, p. 87-96, maio/dez. 1998.

GIL, Antonio Carlos. Técnicas de pesquisa em economia e elaboração de monografias. 3. ed. São Paulo: Atlas, 2000.

IGLESIAS, Enrique V. El papel del Estado y los Paradigmas Económicos en América Latina. Revista de La CEPAL, Santiago de Chile: Comisión Económica para América Latina y el Caribe (CEPAL) / ONU, n. 90, dic. 2006.

IPARDES. Leituras regionais: Mesorregiões Geográficas Paranaenses: Sumário Executivo. Instituto Paranaense de Desenvolvimento Econômico e Social. Curitiba: IPARDES, 2004.

IPARDES. Tipologia dos municípios paranaenses segundo indicadores socioeconômicos e demográficos. Curitiba: Instituto Paranaense de Desenvolvimento Econômico e Social, 2003.

IPEADATA. Dados econômicos. Disponível em: <www.ipeadata.gov.br>. Acesso em: 15 abr. 2008.

LERDA, Juan Carlos; ACQUATELLA, Jean; GÓMEZ, José Javier. Coordinación de políticas públicas: desafíos y oportunidades para una agenda fiscal-ambiental. In: ACQUATELLA, Jean; BÁRCENA, Alicia (Eds.). Política fiscal y medio ambiente: bases para una agenda común. Santiago de Chile: Comisión Económica para América Latina y el Caribe (CEPAL)/ONU, 2005. p. 65-88.

LOWI, T. Four systems of policy, politics, and choice. Public Administration Review, 32, p. 298-310, 1972.

MAGALHÃES FILHO, F. de B. B. de. Da construção ao desmanche: análise do projeto paranaense de desenvolvimento. 1999. Tese (Doutorado em Sociologia) - Faculdade de Filosofia, Letras e Ciências Humanas, Universidade de São Paulo, São Paulo.

MENICUCCI, T. A implementação da reforma sanitária da: a formulação de uma política. In: HOCHMAN, G.; ARRETCH, M.; MARQUES, E. (org.). Políticas Públicas no Brasil. Rio de Janeiro: Fiocruz, 2007. p. 303-326.

MONTEIRO NETO, A. Intervenção estatal e desigualdades regionais no Brasil: contribuições ao debate contemporâneo. Texto para discussão 1229. IPEA - Instituto de Pesquisa Econômica Aplicada. Brasília, novembro 2006.

OLIVEIRA, J. A. P. de. Desafios do planejamento em Políticas Públicas: diferentes visões e práticas. Revista de Administração Pública - RAP, Rio de Janeiro 40(2): 273-88, mar./abr. 2006. 
Implementing environmental policies in developing countries through descentralization: the case of protected areas in Bahia, Brazil, World Development, v. 30, n. 10, p. 1713-1736, 2002.

PRITCHETT, L. The Tyranny of Concepts CUDIE (Cumulated, Depreciated Investment Effort) Is Not Capital. POLICY RESEARCH WORKING PAPER 2341. The World Bank Development - Research Group Poverty and Human Resources. NW, Washington/ DC, USA, May 2000.

REZENDE, D.A.; CASTOR, B.V.J.Planejamento estratégico municipal: empreendedorismo participativo nas cidades, prefeituras e organizações públicas. 2. ed. Rio de Janeiro: Brasport, 2006.

REZENDE, F.C. Por que falham as reformas administrativas? Rio de Janeiro: FGV, 2004.

RODRIGUES, G. Democracia e partidos políticos: os gastos públicos municipais como instrumento de análise político-ideológico. In: HOCHMAN, G.; ARRETCH, M.; MARQUES, E. (org.). Políticas Públicas no Brasil. Rio de Janeiro: Fiocruz, 2007. p. 275-302.

SECRETARIA de Estado do Planejamento e Coordenação Geral (SEPL). Paraná Plano Plurianual 2008 a 2011. Lei n. 15.757, de 27 de dezembro de 2007. Paraná, 2007.

Plano de Governo do Paraná 2003 a 2006. Desenvolvimento Sustentável e Inclusão Social. Curitiba, 2003.
SILVA, C.L. Investimento estrangeiro direto: da dependência à globalização. Rio de Janeiro: Fundo de Cultura, 2005.

Desenvolvimento sustentável: um conceito multidisciplinar. In: SILVA, C.L.; MENDES, J.T.G. (Org.). Reflexões sobre o desenvolvimento sustentável: agentes e interações sob a ótica multidisciplinar. 1. ed. Rio de Janeiro: Vozes, 2005. p. 11-40.

SILVA, Christian Luiz da; MICHON JUNIOR, Wilian. Desenvolvimento socioeconômico local e reestruturação produtiva paranaense na década de 1990. Interações, Campo Grande, v. 9, n. 1, p. 29-43, 2008.

SOTO TORRES, M. Dolores; FERNÁNDEZ LECHÓN, Ramón. Feedback Process in Economic Growth: relations between hours worked and labour productivity. Proceeding... Conference System Dinamics, p. 1-20. Disponível em: <www.systemdynamics.org/conferences/2006/ proceed/papers/soto-170.pdf>. Acesso em: dez. 2006. SOUZA, C. Estado da arte da pesquisa em políticas públicas. In: HOCHMAN, G.; ARRETCH, M.; MARQUES, E. (org.). Políticas Públicas no Brasil. Rio de Janeiro: Fiocruz, 2007. p. 65-86.

SOUZA, M.L. Algumas notas sobre a importância do espaço para o desenvolvimento social. Território, Rio de Janeiro: LAGET-UFRJ/Garamont, n. 3, jul./dez. 1997. 


\section{Apêndice 1 - indicadores socioeconômicos selecionados e respectiva correlações}

Observa-se que a apresentação da matriz de correlação com todos os indicadores não foi apresentada por dificuldade de representação da mesma, pois possui 95 linhas e 95 colunas. Desta forma, as tabelas $1 \mathrm{~A}$ a $4^{\mathrm{a}}$ representam a síntese dos dados tratados e utilizados para reflexão.
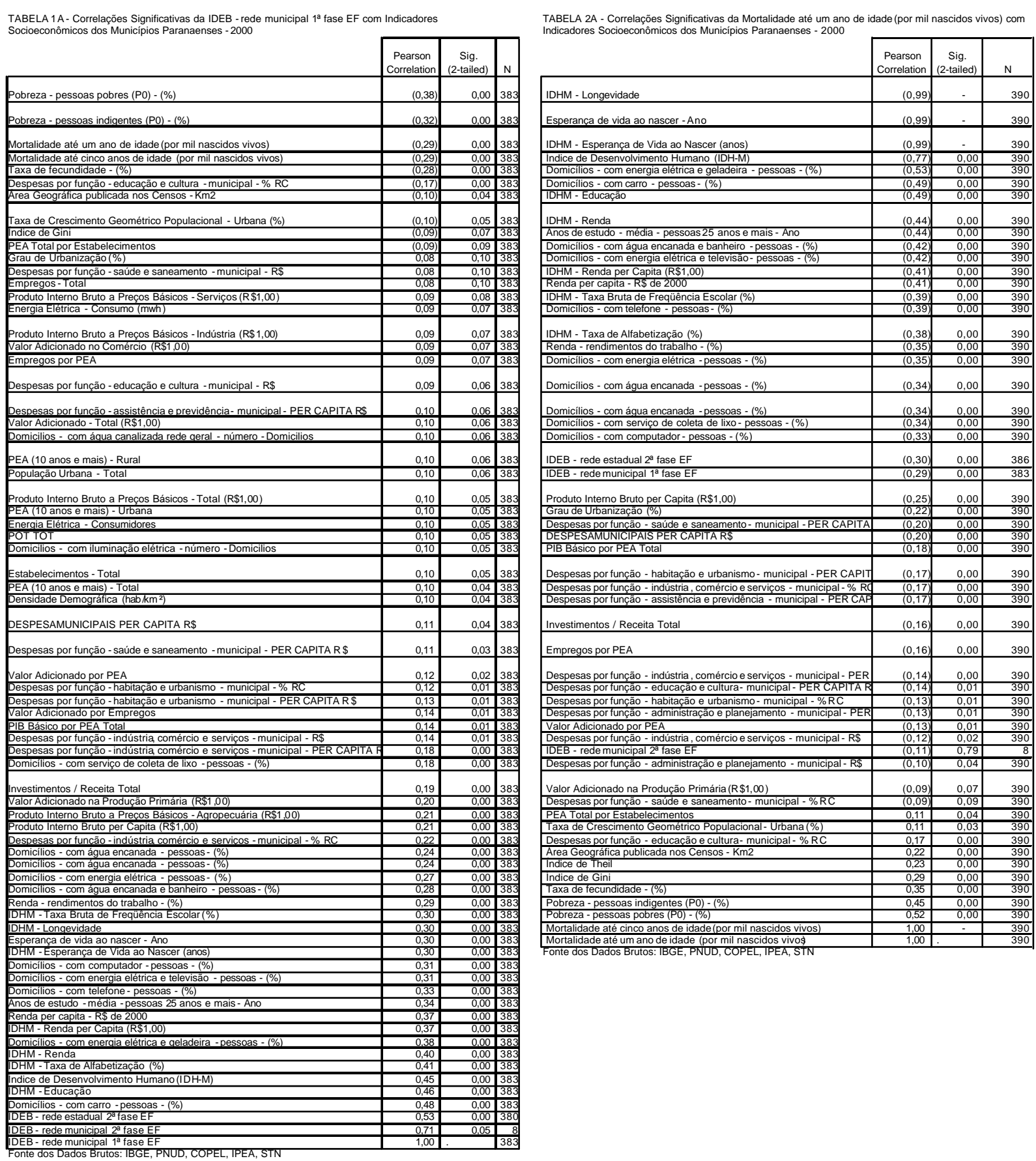